\title{
PyDII: A Python Framework for Computing Equilibrium Intrinsic Point Defect Concentrations and Extrinsic Solute Site Preferences in Intermetallic Compounds
}

\author{
Hong Ding ${ }^{\mathrm{a}, *}$, Bharat Medasani ${ }^{\mathrm{b}, *}$, Wei Chen ${ }^{\mathrm{c}}$, Kristin A. Persson $^{\mathrm{c}}$, Maciej Haranczyk ${ }^{\mathrm{b}}$, \\ Mark Asta ${ }^{\mathrm{a}}$ \\ ${ }^{a}$ Department of Materials Science and Engineering, University of California, Berkeley, CA 94720, USA \\ ${ }^{b}$ Computational Research Division, Lawrence Berkeley National Laboratory, Berkeley, CA 94720, USA \\ ${ }^{c}$ Environmental Energy Technologies Division, Lawrence Berkeley National Laboratory, Berkeley, CA 94720, USA
}

\begin{abstract}
Point defects play an important role in determining the structural stability and mechanical behavior of intermetallic compounds. To help quantitatively understand the point defect properties in these compounds, we developed PyDII, a Python program that performs thermodynamic calculations of equilibrium intrinsic point defect concentrations and extrinsic solute site preferences in intermetallics. The algorithm implemented in PyDII is built upon a dilute-solution thermodynamic formalism with a set of defect excitation energies calculated from first-principles density-functional theory methods. The analysis module in PyDII enables automated calculations of equilibrium intrinsic antisite and vacancy concentrations as a function of composition and temperature (over ranges where the dilute solution formalism is accurate) and the point defect concentration changes arising from addition of an extrinsic substitutional solute species. To demonstrate the applications of PyDII, we provide examples for intrinsic point defect concentrations in $\mathrm{NiAl}$ and $\mathrm{Al}_{3} \mathrm{~V}$ and site preferences for $\mathrm{Ti}$, Mo and $\mathrm{Fe}$ solutes in $\mathrm{NiAl}$.
\end{abstract}

\section{PROGRAM SUMMARY}

Program title: PyDII

Licensing provisions: The MIT License

Program obtainable from: https://github.com/pydii/pydii

Distribution format: Git repository

Programming language: Python

Computer: Any computer with a Python interpreter

RAM: Problem dependent

External routines/libraries: NumPy [1], Sympy [2], and Pymatgen [3]

Nature of problem: Equilibrium intrinsic point defect concentrations and solute site preferences in intermetallic compounds

Running time: Problem dependent

Additional comments: This article describes version 1.0.0.

(C) 2014 Published by Elsevier Ltd.

Keywords: Intrinsic Point Defect, Extrinsic Solute Site Preference, Intermetallic Compound, Python

\footnotetext{
${ }^{*}$ Corresponding authors: H. Ding (hongding @ berkeley.edu) and B. Medasani (bkmedasani@ @lbl.gov). These authors contributed equally to this work.
} 


\section{Introduction}

Intermetallic compounds are a class of ordered alloy phases, consisting of two or more metallic elements, where each metal species occupies specific crystal lattice sites. The mechanical properties and phase stability of these materials have been actively investigated in a variety of contexts, including the design of materials for high-temperature structural applications. In this context, equilibrium point-defect concentrations, and site preferences for solute additions are important considerations for modeling phase stability and mechanical properties such as creep strength [4, $5,6]$.

In intermetalic compounds that have relatively close-packed crystal structures, the dominant intrinsic point defects are expected to be substitutional antisites and vacancies. The concentration of these intrinsic defects is generally a strong function of the temperature and overall alloy composition. Additionally, solute impurities with sizes similar to those of the constituent atomic species are expected to form substitutional defects, but which sublattice is preferred and whether such substitution triggers formation of additional intrinsic defects are important issues related to phase stability and mechanical behavior. Due to the importance of point-defect properties for the stability and mechanical behavior of intermetallic compounds, the inherent difficulty in their direct experimental measurement presents a significant challenge in the context of alloy design. This section describes the development of a computational tool that is intended to automate the process of performing first-principles prediction of point defect concentrations as a function of temperature and composition.

The purpose of developing an automated computational tool, here referred to as PyDII (Python framework for Defects In Intermetallics), is to provide a user-friendly toolkit for predicting these properties based on density functional theory (DFT) calculations. PyDII uses the grand-canonical, dilute-solution thermodynamic formalisms developed in Ref. [7] to predict intrinsic point defect equilibria and solute site preference. The scripts to generate the inputs and parse the results from DFT computational work are based on the open Python Materials Genomics (pymatgen) library [3]. PyDII is intended to be useful for the research community interested in studying the intrinsic intermetallic defects and conducting high-throughput screening of solute additions to produce desired changes in intrinsic properties. The following serves as a concise overview of the method and a user guide for this computational tool.

The rest of this manuscript is organized as follows: Section 2 describes the computational algorithm and workflows of PyDII. The implementation details are described in Section 3, with discussion of technical requirements, input and output of PyDII. Several examples are illustrated in Section 4, followed with a summary in Section 5.

\section{Methodology}

\subsection{Intrinsic Point Defect Concentrations}

We consider an intermetallic compound composed of $M$ distinct elements, with a crystal structure containing a basis of $N$ atomic sites per unit cell. Among these $N$ sites, there are $n$ symmetrically distinct positions having a multiplicity (number of equivalent sites) of $\lambda(p)$, where $p \in[1, \ldots, n]$. The site occupation, $c_{i}(p)$, with $i \in[1, \ldots, M]$, is defined as 1 if lattice site $p$ is occupied by element $i$, or 0 otherwise. For a perfectly ordered stoichiometric structure, $c_{i}(p)$ is given by $c_{i}^{0}(p)$ and $N=\sum_{i=1}^{M} \sum_{p=1}^{n} \lambda(p) c_{i}^{0}(p)$. In general, for any composition, the total number of sites occupied by the $i^{\text {th }}$ element, $N_{i}$, is given by the sum over all lattice occupation values, i.e. $N_{i}=\sum_{p=1}^{n} \lambda(p) c_{i}(p)$.

To predict the equilibrium concentration of point defects, we make use of the grand-canonical, dilute-solution model thermodynamic formalism described in Ref. [7], in which a first-order low-temperature expansion yields the following form for the grand potential $\Omega$ (assuming zero stress):

$$
\Omega=E^{0}-\sum_{i} \mu_{i} \sum_{p} \lambda(p) c_{i}^{0}(p)-k_{B} T \sum_{p} \lambda(p) \sum_{\epsilon} \exp \left\{-\left[\delta E^{\epsilon}(p)-\sum_{i} \mu_{i} \delta c_{i}^{\epsilon}(p)\right] / k_{B} T\right\} .
$$

Here, $\mu_{i}$ is the chemical potential of element $i, k_{B}$ is Boltzmann's constant and $T$ is temperature. $E^{0}$ denotes the ground-state energy for a perfectly ordered stoichiometric alloy. For each possible defect $\epsilon$ at lattice site $p$, the corresponding changes in energy and site-composition are represented by $\delta E^{\epsilon}(p)$ and $\delta c_{i}^{\epsilon}(p)$, respectively. Using the same terminology as in Ref. [7], hereafter, $\delta E^{\epsilon}(p)$ and $\delta E^{\epsilon}(p)-\sum_{i} \mu_{i} \delta c_{i}^{\epsilon}(p)$ will be referred as the defect "excitation energy" and defect formation energy, respectively. From Eq. (1), the following relation can also be derived:

$$
\left\langle c_{i}(p)\right\rangle=c_{i}^{0}(p)+\sum_{\epsilon} \delta c_{i}^{\epsilon}(p) \cdot \exp \left\{-\left[\delta E^{\epsilon}(p)-\sum_{j} \mu_{j} \delta c_{j}^{\epsilon}(p)\right] / k_{B} T\right\},
$$


where $\left\langle c_{i}(p)\right\rangle$ denotes the lattice-averaged (ensemble averaged) concentration of element $i$ at site $p$. At a given temperature, Eq. (2) enables us to compute the equilibrium point-defect concentrations for a given set of the chemical potentials, $\mu_{i}$. More commonly, we are interested in knowing the equilibrium point-defect concentrations as a function of the overall alloy composition. Specifically, let $X_{i}=\left\langle N_{i}\right\rangle / \sum_{i}\left\langle N_{i}\right\rangle$ be the mole fraction of species $i$. In general, specifying the $M-1$ independent mole fractions, where $M$ is the number of atom species, provides from Eq. (2) a total of $M-1$ equations for the $M$ values of $\mu_{i}$. In this formalism, the vacancy is treated as an additional species, for which equilibrium is dictated by setting the grand-potential $\Omega$ in Eq. 1 equal to zero (corresponding to thermodynamic equilibrium relative to the number of lattice sites under zero stress conditions). There results then $M$ equations to determine the $M$ values of $\mu_{i}$, from which the equilibrium point defect concentrations $\left\langle c_{i}(p)\right\rangle$ can be determined from Eq. (2).

The left panel of Fig. 1 is the flow chart describing the calculations of constitutional and thermal defect concentrations. The intermetallic compound information like crystal structure, symmetrically distinct sites, and stoichiometric concentration are derived from either experimental or computational entries in a database. The defect module of Pymatgen is used to generate the proper-size supercell and defect structures and corresponding DFT settings. After the DFT calculations are finished, the post processing codes are used first to obtain the defect "excitation energy" and then to solve for the chemical potential of each species at a given temperature and composition based on the multi-dimensional Newton method implemented in Sympy [2]. The resulting values of the chemical potentials are substituted into Eq. (2) to obtain the corresponding defect concentrations.

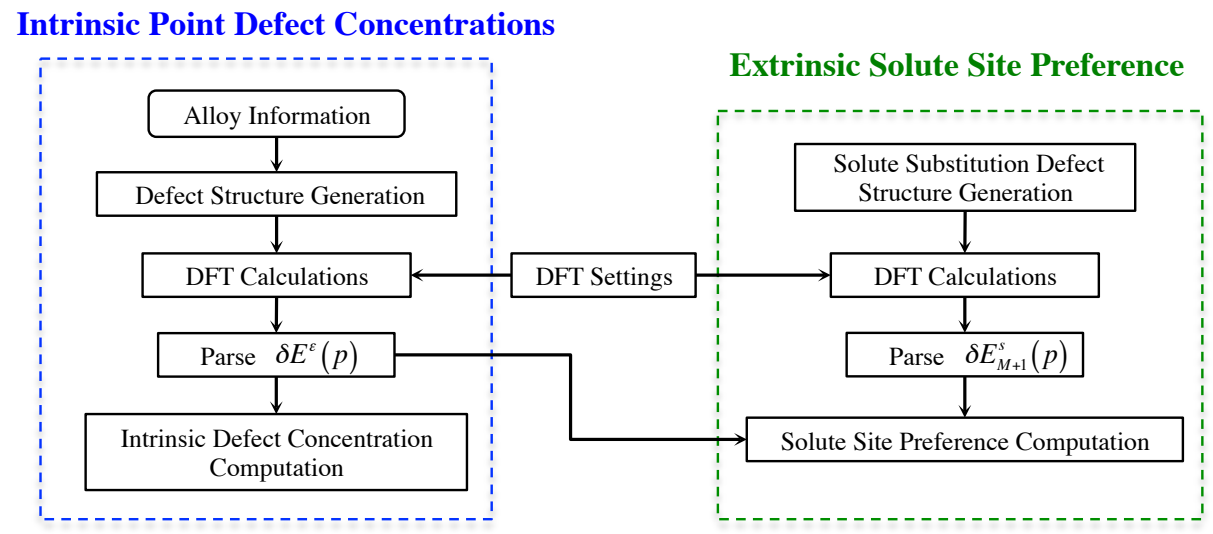

Figure 1: Flow chart describing the workflow for the intrinsic point-defect concentration and extrinsic solute site preference computation.

\subsection{Extrinsic Solute Site Preference}

The extrinsic solute site preference calculation is a simple extension to the above mentioned formalism. Given an external element $(M+1)$ in the dilute limit is introduced into the compound, the site preference of the solute over the $N$ different lattice sites can be considered within the same computational framework. In addition to the vacancy and antisite defect computations that have been done before, another set of $N$ defect structures representing the substitutional defects of the external element on each lattice site are established and calculated, by regarding the external element as the element $M+1$ in the compound. The external element introduces one extra chemical potential into Eq. (2), requiring an additional equation to compute the chemical potentials. The extra constraint can be introduced by imposing a small but fixed amount of the mole fraction (like $0.5 \%$ or $1 \%$ ) for the external element in the alloy. Herein, the introduction of this $(M+1)^{\text {th }}$ constraint into the system would yield another set of chemical potentials for the alloy at a given temperature and composition. Similar to the previous defect concentration computation, by substituting the chemical potentials into Eq. (2), the corresponding defect concentration can be derived, and the site preference can be determined by comparing the solute occupation over each lattice sites, i.e. $\left\langle c_{M+1}(p)\right\rangle /\left\langle c_{M+1}\left(p^{\prime}\right)\right\rangle$. The workflow for the calculations of solute site preference computation is presented in the right panel of Fig. 1, where an additional $n$ external element substitution structure calculations would be set for the defect structure computations. 


\section{Running the code}

In this section, we will describe the basic procedure for performing the required calculations in PyDII. The software developed for this purpose is in Python, so the user proficient in python can customize the software according to their needs, e.g., if interstitial sites are to be included. The software has a dependency on NumPy [1], SymPy [2], and Pymatgen [3] python packages. To determine the intermetallic defect property, the most important input is the crystal structure, which gives basic information about symmetry, lattice site, stoichiometric concentration etc. The Materials Project [8] has provided free access to crystal structure information of many intermetallics through its Materials Application Programming Interface (MAPI) [3]. In the Materials Project database, each structure is assigned a unique id called mpid (Materials Project Id). In what follows, the B2-NiAl structure, with a mpid of mp-1487, will be taken as an example for running these defect property calculations. In the commands presented in this article, the user is expected to have access to the Materials Project. However, its not a strict requirement and one can specify the structure (in standard formats such as CIF, CSSR and etc.) in place of mpid by small changes of the scripts.

\subsection{Equilibrium Intrinsic Point Defect Concentrations}

The procedure to calculate equilibrium intrinsic point-defect concentrations starts with generating the defect supercells and the bulk supercell using the defect structure generator within pymatgen. Then the input files for the Vienna Ab-initio Simulation Package (VASP) [9, 10], a first-principles DFT package are generated using the VASP IO module of pymatgen [3]. The two steps are combined into a single command:

$$
\begin{aligned}
& \triangleright \text { gen_def_structure } \quad \text {--mpid } \quad\langle\text { mpid }\rangle \\
& {\left[\begin{array}{lll} 
& \text {--mapi_key } & \langle\text { mapi_key } \\
{\left[\begin{array}{ll}
\text {--cellmax } & \langle\text { max_no_atoms_in_supercell }\rangle
\end{array}\right]}
\end{array}\right.}
\end{aligned}
$$

This command creates the corresponding "mpid" folder which contains one bulk and several defect structure folders. The 〈mapi key〉 tag is the API key for accessing the Materials Project database [11]. The cellmax parameter is the maximal number of atoms in the defect supercell. If not given, a default value of 128 is used according to our convergence tests for metal systems [12,13]. Among the subfolders in the main "mpid" folder, one "bulk" subfolder would contain the files for the supercell calculation of the energy of the undefected reference crystal structure, and the other subfolders can be classified into two categories according to the type of defects: vacancy and antisite. Each defect supercell calculation is put in one subfolder; for the case of $\mathrm{NiAl}$ (mp-1487) where there are two distinct lattice sites, there would be four defect folders, corresponding to one antisite and one vacancy defect on each sublattice:

\begin{tabular}{ll}
\hline Defect Type & Folder Name \\
\hline $\mathrm{Vac}_{\mathrm{Al}}$ & vacancy_1_mult-1_sitespecie-Al \\
$\mathrm{Vac}_{\mathrm{Ni}}$ & vacancy_2_mult-1_sitespecie-Ni \\
$\mathrm{Ni}_{\mathrm{Al}}$ & antisite__mult-1_sitespecie-Al_subspecie-Ni \\
$\mathrm{Al}_{\mathrm{Ni}}$ & antisite___mult-1_sitespecie-Ni_subspecie-Al \\
\hline
\end{tabular}

Besides the structure file (POSCAR) in each folder, other files (e.g. INCAR, KPOINTS, POTCAR) with computation details can be automatically generated according to user's settings in pymatgen. The settings chosen for each of the files should be tested thoroughly.

Provided that all the DFT calculations including defect and bulk structures are successfully completed, the next step is to parse the results to obtain the defect "excitation energies", $\delta E^{\epsilon}(p)$, which are simply the energy differences between the undefected reference crystal structure and a given defect structure. To accomplish this task, a typical command-line would be

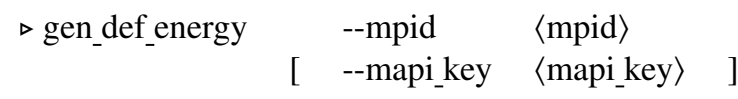


Once the command has successfully parsed the energies for all the defects, it replies with:

\section{DFT calculations successful for $\langle$ mpid $\rangle$}

The output file "〈mpid $\rangle$ _raw_defect_energy.json" contains the defect "excitation energies", as well as other structure information necessary to calculate the defect concentrations. The data in the json file is used to derive the intrinsic point-defect concentrations at a given temperature as a function of the concentration of the system, around the stoichiometric concentration (default is $\pm 0.5 \%$ ). This is accomplished by typing the command:

$$
\triangleright \text { gen_def_profile } \quad \begin{array}{lll} 
& \text {--mpid } & \langle\text { mpid }\rangle \\
{\left[\begin{array}{lll}
\text {--temp } & \langle\mathrm{T}\rangle
\end{array}\right]}
\end{array}
$$

Here, temperature parameter $\langle\mathrm{T}\rangle$ indicates the temperature of interest (in units of $\mathrm{K}$ ), with a default value of $1000 \mathrm{~K}$. This creates three output files, i.e. " $\langle$ mpid $\rangle$ def_concentration.dat", " $\langle$ mpid $\rangle$ _def_energy.dat" and "〈mpid $\rangle$ chem pot.dat", which store the defect concentrations, defect formation energies, and elemental chemical potentials as a function of composition, respectively.

\subsection{Extrinsic Solute Site Preference}

For a given crystal structure, the site preference for a given extrinsic solute species, considered as the $(M+1)^{t h}$ element, can be computed as follows. Firstly, the calculation can be started by the following command :

$$
\begin{aligned}
& \triangleright \text { gen_sol_pref_structure } \quad \text {--mpid } \quad\langle\text { mpid }\rangle \\
& \text {--solute 〈solute element〉 } \\
& \text { [ --mapi key }\langle\text { mapi_key }\rangle \text { ] }
\end{aligned}
$$

This leads to the creation of additional $n$ subfolders inside the main "mpid" folder, corresponding to the configurations where the external solute occupies each of original $n$ sublattice sites. The external solute of interest can be specified by the chemical symbol of the element from the input of 〈solute element $\rangle$.

For example, for the NiAl example the command "gen sit prefence --mpid 1487 --solute Mo' would generate the following two additional subfolders in the "mp-1487" folder for the next sets of DFT calculations:

\begin{tabular}{ll}
\hline Defect Type & Folder Name \\
\hline $\mathrm{Mo}_{\mathrm{Al}}$ & solute_1_mult-1_sitespecie-Al_subspecie-Mo \\
$\mathrm{Mo}_{\mathrm{Ni}}$ & solute_2_mult-1_sitespecie-Ni_subspecie-Mo \\
\hline
\end{tabular}

After finishing the additional $n$ DFT calculations, the solute defect "excitation energies" on each lattice site can be obtained using the command:

$$
\begin{aligned}
& \triangleright \text { gen_sol_def_energy } \quad \text {--mpid } \quad\langle\text { mpid }\rangle \\
& \text {--solute 〈solute element } \\
& \text { [ --mapi key 〈mapi key }{ }_{-} \text {] }
\end{aligned}
$$

The set of solute "excitation energies", $\delta E_{M+1}^{s}(p)$, for the external solute occupying the site $p(p \in[1, \ldots, n])$ is

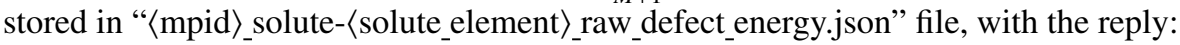

DFT calculations for solute 〈solute_element $\rangle$ on $\langle$ mpid $\rangle$ are successful 
Along with the previous output file "〈mpid $\rangle_{-}$raw_defect_energy.json", the site preference of the external solute can be analyzed by typing:

$$
\begin{aligned}
& \triangleright \text { gen_sol_site_pref } \quad \text {--mpid }\langle\text { mpid }\rangle \\
& \text {--solute 〈solute element〉 } \\
& \text { [ --temp }\langle\mathrm{T}\rangle \text { - }
\end{aligned}
$$

Here, the temperature parameter also has a default value of $1000 \mathrm{~K}$. This command outputs the solute concentrations on each lattice site and and the corresponding intrinsic defect concentrations as a function of stoichiometric

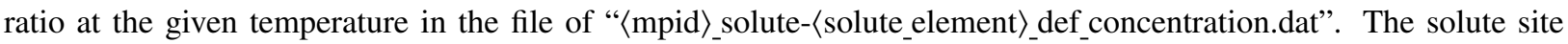
occupation ratios can be derived from the output results subsequently.

\section{Example}

PyDII scripts were initially benchmarked based on the first-principles energies reported in Ref. [7] for $\mathrm{Al}_{3} \mathrm{Sc}$ and shown to be able to reproduce earlier results. Here, three other sets of examples are presented; the first two show the equilibrium intrinsic defect computations for $\mathrm{NiAl}$ (mp-1487) and $\mathrm{Al}_{3} \mathrm{~V}$ (mp-2554) and the third example demonstrates the site preference calculations of Ti, Mo and Fe solutes in the NiAl (mp-1487) crystal structure.

\subsection{Intrinsic Point Defect Concentrations}

(a) $\mathrm{NiAl}$

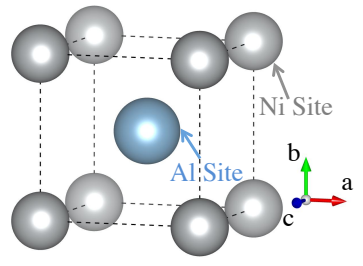

(b) $\mathrm{Al}_{3} \mathrm{~V}$

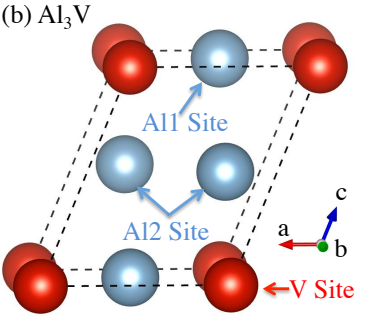

Figure 2: Crystal structure of (a) $\mathrm{NiAl}$ with one $\mathrm{Ni}$ and one $\mathrm{Al}$ site (b) $\mathrm{Al}_{3} \mathrm{~V}$ with two symmetrically distinct $\mathrm{Al}$ and one $\mathrm{V}$ site.

\subsubsection{NiAl (mp-1487)}

The well-studied B2-NiAl (mp-1487) crystal structure, as shown in Fig. 2(a), is used to verify that our codes produce output consistent with previously published calculations $[14,15,16,17]$. In the $\mathrm{B} 2-\mathrm{NiAl}$ structure, $\mathrm{Ni}$ and $\mathrm{Al}$ atoms occupy the corner and center lattice sites of a cubic cell with the $\mathrm{Pm} \overline{3} \mathrm{~m}$ (221) space group. Four different defect types are considered in the calculation: $\mathrm{Vac}_{\mathrm{Al}}, \mathrm{Vac}_{\mathrm{Ni}}, \mathrm{Al}_{\mathrm{Ni}}$ and $\mathrm{Ni}_{\mathrm{Al}}$. Equilibrium concentrations of these defects at the temperature of $T=1000 \mathrm{~K}$ are plotted in Fig. 3(a). As found in previous calculations [14, 15, 16, 17] and deduced from experimental measurements [18], the current results show that the dominant point defect for Ni-rich concentrations is $\mathrm{Ni}_{\mathrm{Al}}$, while for Ni-rich compositions it is $\mathrm{Vac}_{\mathrm{Ni}}$. The relative order of magnitude for the four defect concentrations is in agreement with results reported in previous calculations based on DFT [14, 17] and classical interatomic potential model $[15,16]$ calculations. Differences within a factor of two are found for the dominant defect concentrations within the composition region between between the present calculations and previously published results. These differences are reasonable given the different energy methods and statistical-mechanical frameworks used in the previously published work. 


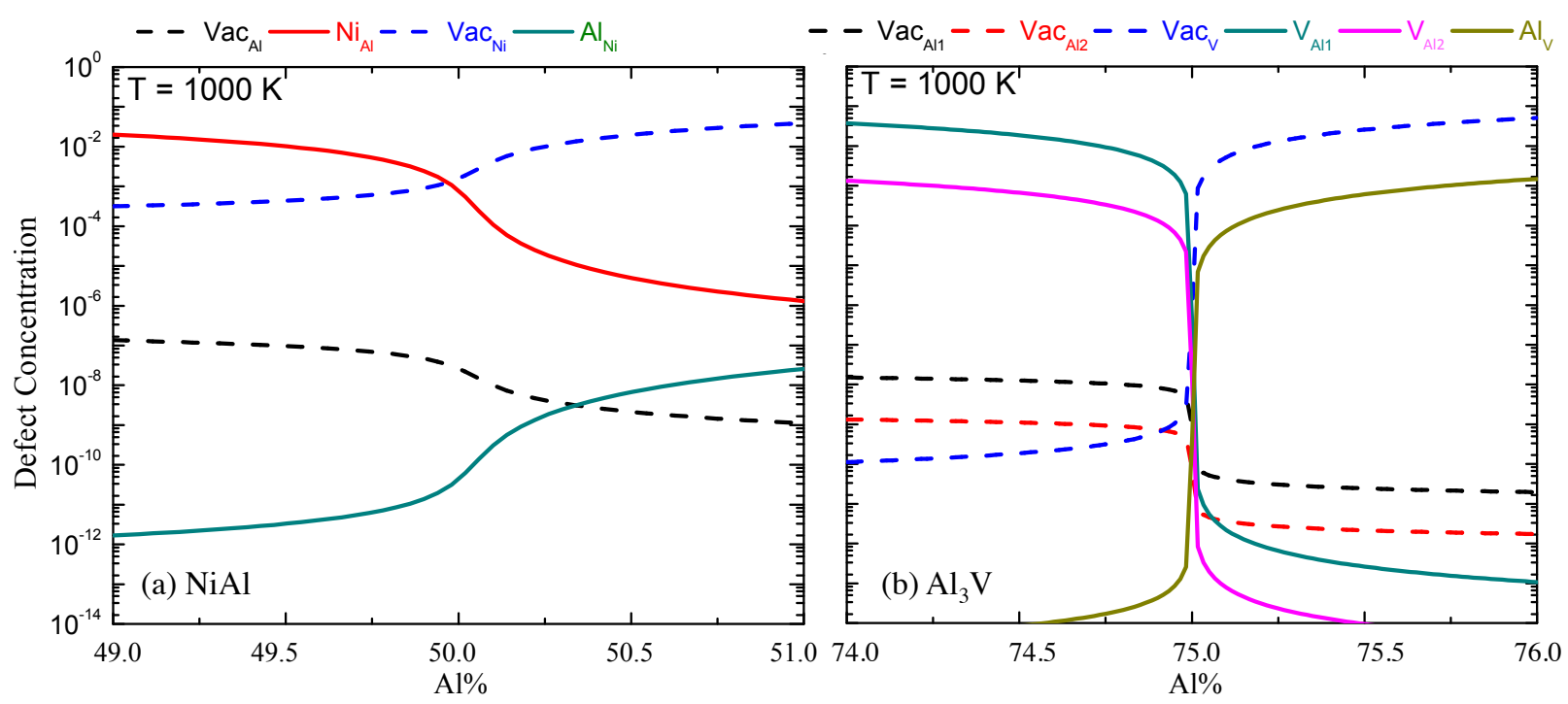

Figure 3: Equilibrium intrinsic point-defect concentrations in (a) $\mathrm{NiAl}$ and (b) $\mathrm{Al}_{3} \mathrm{~V}$ at $\mathrm{T}=1000 \mathrm{~K}$ as a function of the mole fraction of $\mathrm{Al}$.

\subsection{2. $\mathrm{Al}_{3} V(\mathrm{mp}-2554)$}

The current code also enables the distinction between symmetrically inequivalent sites and this is demonstrated with $\mathrm{Al}_{3} \mathrm{~V}$ (mp-2554). The $\mathrm{Al}_{3} \mathrm{~V}$ crystal structure has the I4/mmm (139) space group with two symmetrically distinct Al lattice sites and one $\mathrm{V}$ site in a primitive cell, as shown in Fig. 2(b). The symmetrically inequivalent Al sites warrant additional calculations leading to three vacancy and three antisite calculations. The constitutional and thermal defect concentration profiles for $\mathrm{Al}_{3} \mathrm{~V}$ around the ideal stoichiometry are plotted in Fig. 3(b). Our calculations indicate that for Al-poor concentrations, the majority defects in the structure are $\mathrm{V}$ antisites on the Al sublattices, while for Al-rich concentrations, the dominant defects are vacancies on the $\mathrm{V}$ sublattices.

A comparison between Al1 and Al2 sites shows that the excess V preferentially occupies Al1 site, and the antisite defect concentration $\mathrm{V}_{\mathrm{Al} 1}$ is about one order of magnitude higher than that of $\mathrm{V}_{\mathrm{Al} 2}$. These calculations demonstrate the significant antisite defect property differences between the two symmetrically distinct $\mathrm{Al}$ sites in the $\mathrm{Al}_{3} \mathrm{~V}$ crystal structure. 


\subsection{Extrinsic Solute Site Preference}

4.2.1. Solute Ti, Mo and Fe in NiAl (mp-1487)

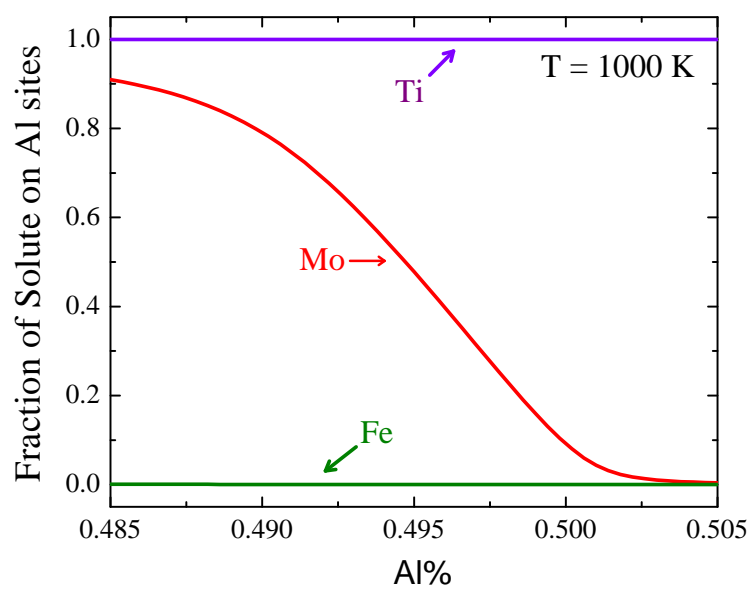

Figure 4: Calculated fraction of solute $\mathrm{X}(\mathrm{X}=\mathrm{Ti}$, Mo or $\mathrm{Fe})$ occupying the $\mathrm{Al}$ sublattice in $\mathrm{Ni}_{x} \mathrm{Al}_{0.99-x} \mathrm{X}_{0.01}$ alloys at $\mathrm{T}=1000 \mathrm{~K}$ as a function of the mole fraction of Al.

Solute site preference calculation examples are presented for solutes Ti, Mo and Fe in NiAl (mp-1487). Fig. 4 shows the calculated fraction of solute on the Al sublattice as a function of Al concentration. Within the composition region considered, the present examples feature three type of elements (i) strong site preference of Ti to Al sublattice, (ii) composition-dependent site preference of Mo and (iii) strong site preference of Fe to Ni sublattice. The behavior of these three solute species in $\mathrm{NiAl}$ is consistent with the previous calculations by Jiang [19].

\section{Summary}

PyDII implements a dilute-solution thermodynamic formalism to predict the intrinsic vacancy and antisite pointdefect concentrations and extrinsic solute site preferences in intermetallic compounds from first-principles DFT calculations. We present a detailed user guide here describing how to run the code, and showcase studies to demonstrate how the program can be used for systematic explorations of point defect properties in intermetallics.

\section{Acknowledgments}

This work was intellectually led by the Materials Project Center, supported by the Office of Basic Energy Sciences (BSE) of the U.S. Department of Energy (DOE) under Grant No. EDCBEE. H Ding was supported by the U.S. DOE, Office of Fossil Energy, under Grant No. DE-FG000568, while all other authors were supported by the Materials Project Center. This work used resources of the National Energy Research Scientific Computing Center, supported by the BSE of the U.S. DOE under Contract No. DE-AC02-05CH11231. The authors gratefully acknowledge helpful discussions with A. Canning, A. Jain and S. P. Ong.

\section{Reference}

[1] Numpy Developers, http://numpy.org/.

[2] Sympy Development Team, http://sympy.org/. 
[3] S. P. Ong, W. D. Richards, A. Jain, G. Hautier, M. Kocher, S. Cholia, D. Gunter, V. L. Chevrier, K. A. Persson, G. Ceder, Python Materials Genomics (pymatgen) : A Robust, Open-Source Python Library for Materials Analysis, Computational Materials Science 68 (2013) $314-319$.

[4] N. Stoloff, C. Liu, S. Deevi, Emerging Applications of Intermetallics, Intermetallics 8 (9) (2000) 1313-1320.

[5] G. Frommeyer, R. Fischer, J. Deges, R. Rablbauer, A. Schneider, Apfim investigations on Site Occupancies of the Ternary Alloying Elements $\mathrm{Cr}, \mathrm{Fe}$, and Re in NiAl, Ultramicroscopy 101 (2) (2004) 139-148.

[6] A. Lasalmonie, Intermetallics: Why is it so Difficult to Introduce them in Gas Turbine Engines?, Intermetallics 14 (10) (2006) $1123-1129$.

[7] C. Woodward, M. Asta, G. Kresse, J. Hafner, Density of Constitutional and Thermal Point Defects in L12 Al3Sc, Physical Review B 63 (9) (2001) 094103 .

[8] A. Jain, S. P. Ong, G. Hautier, W. Chen, W. D. Richards, S. Dacek, S. Cholia, D. Gunter, D. Skinner, G. Ceder, et al., Commentary: The Materials Project: A Materials Genome Approach to Accelerating Materials Innovation, APL Materials 1 (1) (2013) 011002.

[9] G. Kresse, J. Hafner, Ab initio Molecular Dynamics for Liquid Metals, Phys. Rev. B 47 (1993) 558.

[10] G. Kresse, J. Hafner, Ab initio Molecular-Dynamics Simulation of the Liquid-Metal-Amorphous-Semiconductor Transition in Germanium, Phys. Rev. B 49 (1994) 14251.

[11] The Materials Project API, http://materialsproject.org/open.

[12] H. Ding, V. I. Razumovskiy, M. Asta, Self Diffusion Anomaly in Ferromagnetic Metals: A Density-Functional-Theory Investigation of Magnetically Ordered and Disordered Fe and Co, Acta Materialia 101 (2014) 130-136.

[13] B. Medasani, M. Haranczyk, A. Canning, M. Asta, Vacancy Formation Energies in Metals: A Comparison of MetaGGA with LDA and GGA Exchange-Correlation Functionals, Computational Materials Science 70 (2015) 96-107.

[14] P. A. Korzhavyi, A. V. Ruban, A. Y. Lozovoi, Y. K. Vekilov, I. Abrikosov, B. Johansson, Constitutional and Thermal Point Defects in B2 NiAl, Physical Review B 61 (9) (2000) 6003.

[15] M. Gururajan, T. Abinandanan, Mean Field Theory of Point Defects in $\beta$-NiAl, Intermetallics 8 (7) (2000) $759-767$.

[16] Y. Mishin, M. Mehl, D. Papaconstantopoulos, Embedded-Atom Potential for B2-NiAl, Physical Review B 65 (22) (2002) 224114.

[17] Q. Xu, A. Van der Ven, First-principles Investigation of Migration Barriers and Point Defect Complexes in B2-NiAl, Intermetallics 17 (5) (2009) 319-329.

[18] A. Bradley, A. Taylor, An x-ray Analysis of the Nickel-Aluminium System, Proceedings of the Royal Society of London. Series AMathematical and Physical Sciences 159 (896) (1937) 56-72.

[19] C. Jiang, Site Preference of Transition-Metal Elements in B2 NiAl: A Comprehensive Study, Acta materialia 55 (14) (2007) $4799-4806$. 